\title{
Brain Gene Expression During REM Sleep Depends on Prior Waking Experience
}

\author{
Sidarta Ribeiro, ${ }^{1}$ Vikas Goyal, ${ }^{2}$ Claudio V. Mello, ${ }^{1}$ and Constantine Pavlides ${ }^{2}$ \\ ${ }^{1}$ Laboratory of Animal Behavior \\ ${ }^{2}$ Laboratory of Neuroendocrinology \\ The Rockefeller University \\ New York, New York, 10021 USA
}

\begin{abstract}
In most mammalian species studied, two distinct and successive phases of sleep, slow wave (SW), and rapid eye movement (REM), can be recognized on the basis of their EEG profiles and associated behaviors. Both phases have been implicated in the offline sensorimotor processing of daytime events, but the molecular mechanisms remain elusive. We studied brain expression of the plasticity-associated immediate-early gene (IEG) $z i f-268$ during $\mathrm{SW}$ and REM sleep in rats exposed to rich sensorimotor experience in the preceding waking period. Whereas nonexposed controls show generalized zif-268 down-regulation during SW and REM sleep, zif-268 is upregulated during REM sleep in the cerebral cortex and the hippocampus of exposed animals. We suggest that this phenomenon represents a window of increased neuronal plasticity during REM sleep that follows enriched waking experience.
\end{abstract}

\section{Introduction}

Mammals spend a considerable portion of their lives sleeping. In addition to its importance for body repose, sleep appears to play a role in the consolidation of daytime memories (Pearlman and Becker 1974; Fishbein and Gutwein 1977; Smith and Butler 1982; Winson 1993; Karni et al. 1994; Hennevin et al. 1995; Smith 1996). Electrophysiological studies revealed that hippocampal place cells are reactivated during slow wave (SW) sleep (Pavlides and Winson 1989; Wilson and McNaughton 1994) in a manner that recapitulates the neuronal firing sequences that occur during the preceding wakefulness (WK) period (Skaggs and McNaughton 1996). Recent evidence also indicates

${ }^{1}$ Corresponding author. that the firing profiles of place cells are altered during the rapid eye movement (REM) sleep that follows exposure to a new environment (G.R. Poe, W.E. Skaggs, C.A. Barnes, and B.L. McNaughton, unpubl.). These observations support the notion that brain reactivation during sleep is important for the processing of sensorimotor information gathered as a result of waking activity. It is unclear, though, by which mechanisms this is accomplished.

Several lines of evidence indicate that the laying down of long-term memories requires long-lasting modification of neuronal connections, most likely through the activation of gene expression programs that lead to neuronal plasticity (Madison et al. 1991; Bliss and Collingridge 1993). A particularly interesting candidate for mediating long-lasting effects of experience on the brain is zif-268 (Milbrandt 1987), an immediate-early gene (IEG) whose expression in the adult brain is highly sensitive to neuronal depolarization (Sukhatme et al. 1988). Zif-268 protein binds to a specific DNA motif present in the promoters of a variety of genes expressed in the nervous system (Christy and Nathans 1989), and its up-regulation is thought to initiate a program of gene regulation leading to neuronal plasticity (Chaudhuri 1997). For instance, Zif-268 has been directly shown to induce the expression of a synapse-specific protein, synapsin II (Petersohn et al. 1995). zif-268 has been linked to the induction of hippocampal long-term potentiation (LTP) (Cole et al. 1989; Wisden et al. 1990; Abraham et al. 1993; Roberts et al. 1996), neuronal morphological changes after exposure to an enriched environment (Wallace et al. 1995), and other plasticity-related phenomena (Nedivi et al. 1993; Kaplan et al. 1995, 1996). Finally, zif-268 is up-regulated in several novelty or learning behavioral paradigms, including two-way active avoidance (Nikolaev et al. 1992), brightness discrimination (Grimm and Tischmeyer 1997), and enriched environment exposure (Wallace et al. 1995). Be-

LEARNING \& MEMORY 6:500-508 @ 1999 by Cold Spring Harbor Laboratory Press ISSN1072-0502/99 \$5.00

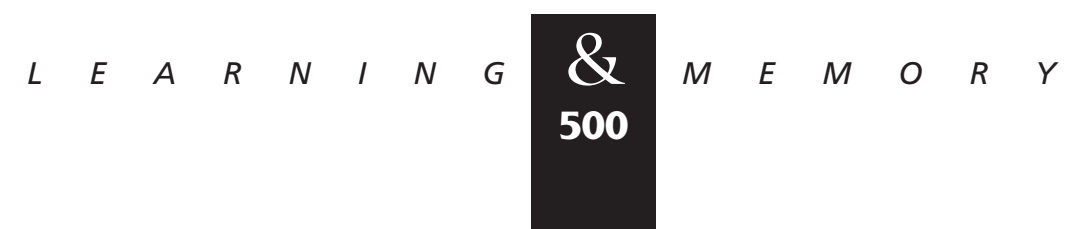


cause zif-268 mRNA levels peak sharply $30 \mathrm{~min}$ after a variety of stimuli (Richardson et al. 1992; Cullinan et al. 1995; Honkaniemi et al. 1995; Mello et al. 1995; Tanaka et al. 1997), its expression can be used for a snapshot assessment of activation throughout the brain (Chaudhuri 1997; Kaczmarek and Chaudhuri 1997; Herdegen and Leah 1998).

In the present study, we tested whether a rich waking sensorimotor experience can modulate $z$ if268 expression levels during ensuing sleep states by assessing the effect of a brief exposure to an enriched environment on zif-268 mRNA levels during $\mathrm{SW}$ and REM sleep in rats.

\section{Materials and Methods}

\section{ELECTRODE IMPLANT AND BASELINE EEG RECORDING}

Adult male Sprague-Dawley rats ( $n=6$ per group, total $n=36$; Charles River) were housed in individual home cages (12/12 hr light/dark schedule; lights on at 9 a.m., food and drink ad libitum). Animals were handled for 15-20 days so as to decrease the stress response to the experimenter, and were then bilaterally implanted with chronic electrodes in the dentate gyrus for EEG recording. After a 3-day postoperative recovery period, baseline EEG was recorded for 10-15 consecutive days to identify typical WK, SW, and REM profiles. Recording was performed inside a soundproof isolation box equipped with one-way mirrors for behavior observation. Surgical procedures, as well as animal care and handling, were in accordance with institutional and National Institutes of Health (NIH) guidelines.

\section{ENRICHED ENVIRONMENT}

The labyrinth was novel to all animals and consisted of four cardboard boxes of different sizes (total area $=2.5 \mathrm{~m}^{2}$ ), interconnected by plastic tubes that allowed free access of animals to all chambers. Platforms and wooden toys were present in the four chambers. Scented corn flakes (five different flavors) were randomly dispersed throughout the labyrinth at semihidden places; two water sources were provided. During the entire stay of animals in the labyrinth, behavior was observed under near-infrared light, to which rats are blind (Neitz and Jacobs 1986).

\section{SLEEP CRITERIA}

REM sleep can be reliably identified in rats by a combination of criteria, including the presence of hippocampal theta rhythm, rhythmic movement of the vibrissae, and highly irregular breathing during sleep; all of these are absent during SW, which has its own characteristic EEG/behavior profile (Vanderwolf 1969; Winson 1974; Timo-Iaria et al. 1990). Animals that were awake for more than 60 min in the recording chamber without sleep episodes were grouped as WK; animals that had at least 3 min of SW sleep but neither theta rhythm nor any behavior associated with REM sleep were grouped as SW; animals that after one or more periods of SW sleep had (1) behavioral signs of REM sleep, and (2) more than 90 sec of theta rhythm within a 5-min interval were grouped as REM. Animals that did not reach criteria for any group were either retested another day (C animals), or discarded from the experiment (EE animals; $n=5$ ).

\section{IN SITU HYBRIDIZATION}

Animals were killed by decapitation and their brains quickly removed and frozen in $-30^{\circ} \mathrm{C}$ methylbutane. Frontal brain sections $(10 \mu \mathrm{m})$ were taken at approximately $2.80 \mathrm{~mm}$ from Bregma (Paxinos and Watson 1986); particular care was taken to ensure that all brains were cut in the same plane. Sections were hybridized with a ${ }^{33}$ P-labeled riboprobe specific for $z i f-268$, following a previously described in situ hybridization protocol (Mello et al. 1992). Test hybridizations were performed so as to optimize stringency conditions; absence of sense-strand hybridization was used as a control for signal specificity. To minimize variability, all sections used for densitometric measurements were hybridized as a single batch. As further specificity controls, adjacent sections were hybridized with riboprobes for krox-20 (Chavrier et al. 1988) and $n g f i-b$ (Milbrandt 1988).

\section{DATA ACQUISITION}

X-ray films exposed to the hybridized sections were imaged with a CCD camera (Hitachi) and densitometric analysis was performed with NIH Image. Adjacent cresyl-stained sections were used as a reference to identify the areas of interest in the X-ray films. Twenty-five different areas were assessed within six major brain subdivisions, as follows: hippocampus [dentate gyrus (DG) and fields CA1, CA2, CA3, and CA4 of Ammon's Horn]; cerebral cortex [retrosplenial granular (RSG), frontal (Fr),

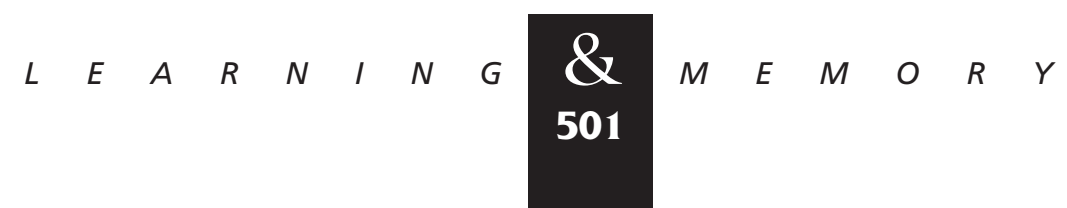




\section{Ribeiro et al.}

parietal (Par), perirhinal (Prh), and piriform (Pir) cerebral cortices]; striatum [caudate-putamen (Cpu)]; amygdala [lateral (La), basolateral anterior (BLA), basolateral ventral (BLV), medial (Me), and posterolateral cortical (PLCo) amygdaloid nuclei]; thalamus [posteroventral (VP), ventral (V), mediodorsal (MD), central medial (CM), and reuniens (Re) thalamic nuclei]; and hypothalamus [dorsomedial (DMD), ventromedial (VMH), arcuate (Arc), and lateral (LH) hypothalamic nuclei]. OD was measured for the entire extent of each area of interest, and averaged across hemispheres. Individual values thus obtained were then normalized to the mean group value obtained for each brain structure. Values for the six major brain divisions were obtained by pooling the normalized values of the corresponding individual brain areas.

\section{STATISTICAL ANALYSIS}

Multivariate analysis of variance (MANOVA) was used to reveal profile differences indicative of state versus condition interactions (StatView 4.0); for the regions in which significant interactions occurred, protected $t$-tests (Bonferroni-Dunn, adjusted for the number of comparisons) were performed to reveal differences across sleep states as well as between $\mathrm{C}$ and EE groups. The same procedure was used for individual brain areas.

\section{Results}

Figure 1 shows the general scheme of our experimental paradigm. To avoid stress-related effects on gene expression (Cullinan et al. 1995;
Jarvis et al. 1995), we subjected animals to an extended period of daily handling and EEG recording prior to the experiment. We also studied spontaneously manifested wake/sleep behaviors, avoiding forced sleep deprivation. The experiment comprised three consecutive phases: an initial exposure to the enriched environment, an intervening period, and a final period of wake/sleep (Fig. 1). Behavior was recorded continuously throughout the entire experiment. At 6 p.m. (lights off) on the day of the experiment, animals belonging to the enriched environment group (EE) were individually placed inside a complex labyrinth for an exposure period that spanned the last $3 \mathrm{hr}$ of the normal wakefulness period, whereas animals from the unexposed control group (C) remained in their home cages. At 9 a.m. (as lights were turned on) animals were placed in their individual home cages for an intervening period of $30 \mathrm{~min}$, and were then transferred to the isolation box in which their hippocampal EEG was continuously recorded. According to the behaviors spontaneously manifested during the recording session, animals were further divided into WK, SW, and REM groups ( $n=6$ per group). After reaching criterion for WK, SW, or REM, animals were left undisturbed for an additional $30 \mathrm{~min}$ (behavior and EEG still monitored) and were then killed. Their brains were processed for $z i f-268$ expression, which was quantified for 25 brain structures within 6 major brain regions: cerebral cortex, hippocampus, striatum, amygdala, thalamus, and hypothalamus.

EE animals remained awake throughout the exposure period, engaging in active exploratory behavior of all the labyrinth chambers. $\mathrm{C}$ animals also
Figure 1: Schematic flowchart of the experimental design. All rats were allowed to get familiarized with the home cage, experimenter, and recording conditions for several days, during which baseline EEG was recorded. On the day of the experiment, animals were either exposed to a complex labyrinth (EE) or kept in their home cages (controls) for the last $3 \mathrm{hr}$ of the waking period, followed by an intervening period of 30 min back in the home cages. The animals were then placed in the recording box for monitoring of wake/sleep states until reaching criterion for WK, SW, or REM; the typical duration of this period was $\sim 80$ min. Thirty minutes after criterion, animals were killed and their brains were processed for zif-268 expression by in situ hybridization.

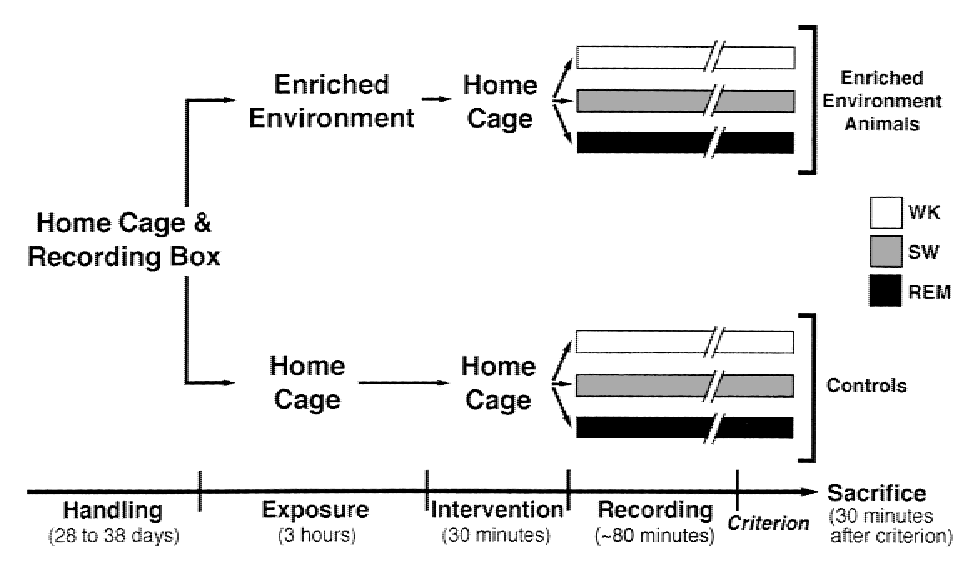


remained awake, displaying grooming and feeding behaviors in addition to a low to moderate degree of locomotion. All animals remained awake during the intervening period, as well as during the last 30 min before sacrifice. The period between exposure to the labyrinth and the moment of sacrifice for the REM group did not differ significantly from those of WK or SW groups (WK $=311 \pm 8$, $\mathrm{SW}=322 \pm 10, \mathrm{REM}=325 \pm 7$; means \pm s.E.M., in minutes). Furthermore, the time spent in REM sleep did not differ significantly between $\mathrm{C}$ and EE animals (148 \pm 40 and $137 \pm 24$, respectively; means s.E.M., in seconds). No qualitative differences between the EEG patterns from EE and C animals were observed within the WK, SW, and REM groups.

zif-268 expression in control animals generally decreased from the WK to the SW and REM groups (Fig. 2, a-a ${ }^{\prime \prime}$ ); this effect was most prominent in the cerebral cortex (Fig. 3). Such a decrease after a single episode of either SW or REM sleep is consistent with previous studies showing that brain expression of IEGs (including zif-268) is downregulated after several hours of sleep, in comparison with levels found in sleep-deprived animals (Pompeiano et al. 1992, 1997; Basheer et al. 1997).

A very different picture emerges when one analyzes animals exposed to an enriched environment, and teases apart the specific contributions of SW and REM: Whereas zif-268 expression decreased from the WK to the SW group (Fig. 2, b to $\left.b^{\prime}\right)$, there was a clear rise from the SW to the REM group (Fig. 2, $\mathrm{b}^{\prime}$ to $\mathrm{b}^{\prime \prime}$ ). This effect was particularly noticeable in the cerebral cortex and in the hippocampus; for both regions, as revealed by MANOVA and protected t-tests (Fig. 3), zif-268 expression levels in EE animals was significantly higher in the REM group than in the SW group. In addition, zif268 levels during REM were higher for EE than for $\mathrm{C}$ animals. Within the cerebral cortex and the hippocampus, the individual structures that most contributed to the effect were respectively the piriform and frontal cortices, and the dentate gyrus (Fig. 4).
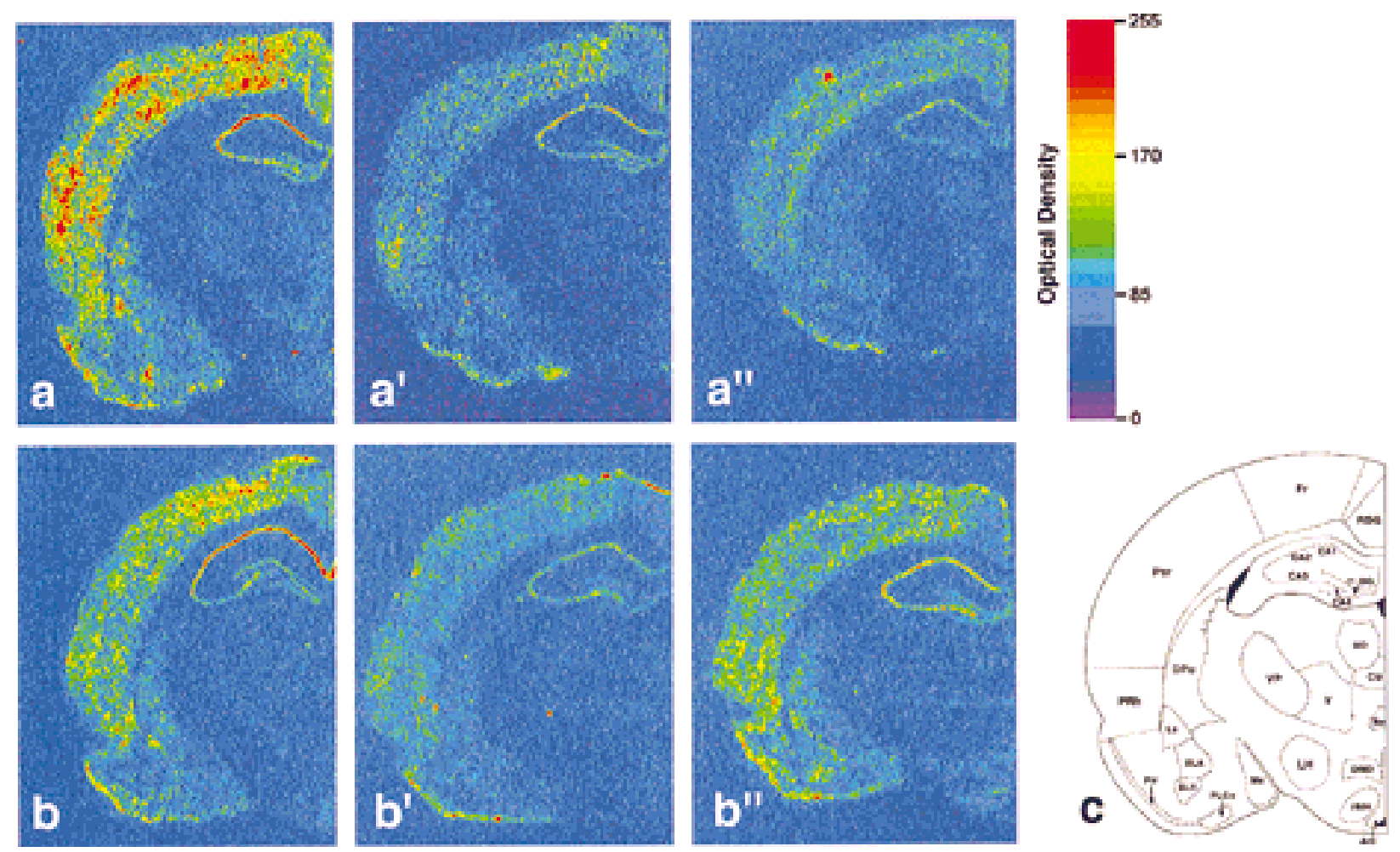

Figure 2: Effect of previous sensorimotor experience on zif-268 brain expression during waking and sleep states. Shown are autoradiograms of brain sections whose gene expression levels best represent the means for each group studied. In controls, zif-268 expression decreased from WK (a) to SW ( $\left.a^{\prime}\right)$ and REM ( $\left.a^{\prime \prime}\right)$. In enriched environment animals, zif-268 levels decreased from WK $(b)$ to SW $\left(b^{\prime}\right)$, but increased from the latter to REM $\left(b^{\prime \prime}\right)$. This effect was particularly noticeable in the cerebral cortex and the hippocampus. (c) Schematic diagram of a frontal section of the rat brain at the level studied; the 25 regions for which expression was quantified are indicated (for abbreviations see Materials and Methods).

$$
\begin{array}{llllllll}
L & E & A & R & N & I & N & G \\
\underset{\mathbf{5 0 3}}{\boldsymbol{\bigotimes}} & M & E & M & O & R & Y
\end{array}
$$




\section{Ribeiro et al.}

Figure 3: Analysis of zif-268 expression during waking and sleep states in six major brain regions; cerebral cortex, hippocampus, striatum, amygdala, thalamus, and hypothalamus. Statistically significant interactions (MANOVA) between expression profiles occurred for the cerebral cortex $(P=0.04)$ and the hippocampus $(P=0.03)$. Whereas zif-268 expression in controls decreased from WK to SW and to REM, in animals exposed to the enriched environment, zif-268 levels decreased from WK to SW but increased from SW to REM. Values on the $y$-axis represent normalized optical density (OD) measurements (means \pm S.E.M.) of $X$ ray film autoradiograms; statistically significant differences (Bonferroni-Dunn) are indicated by one $(P<0.05)$ or two $(P<0.01)$ asterisks. White, gray, and black columns represent, respectively, WK, SW, and REM. Asterisks inside columns indicate state differences between $\mathrm{C}$ and EE groups.
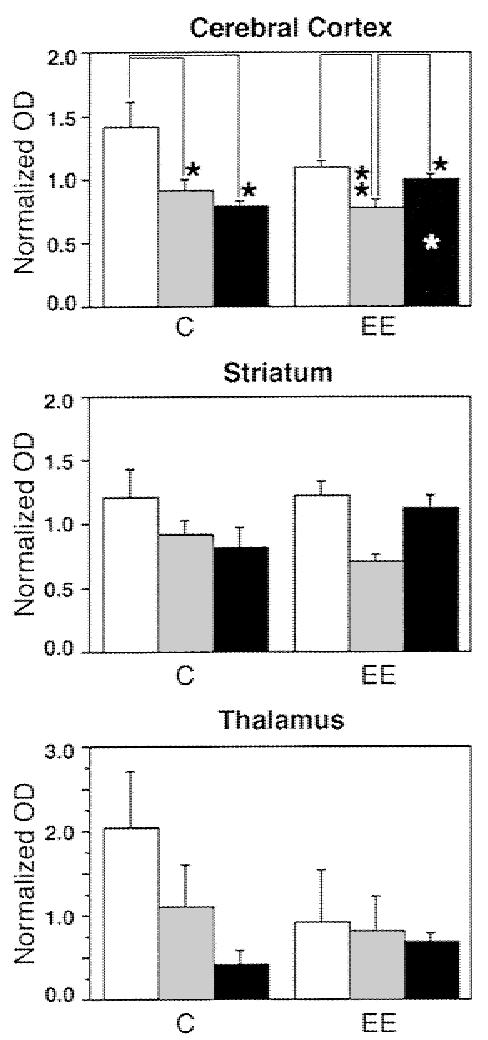
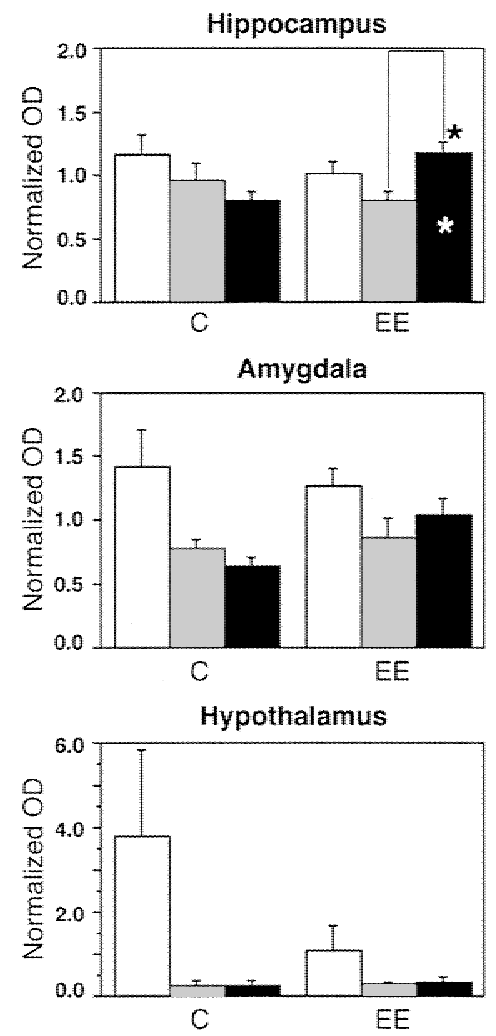

Even though similar trends were observed for the striatum and the amygdala, profile interactions (as revealed by MANOVA; see Materials and Meth- ods) did not reach statistical significance in these regions; for the thalamus and the hypothalamus, no up-regulation was observed (Fig. 3). The effects

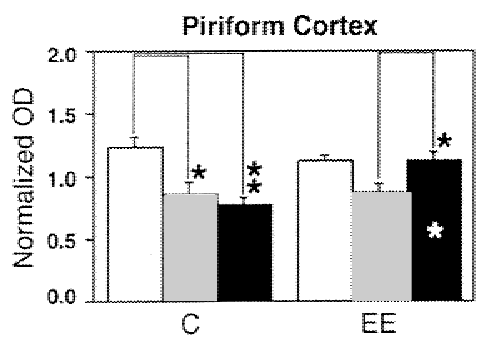

Field CA1 of Ammon's Horn

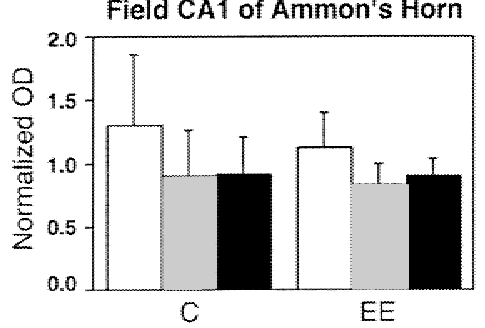

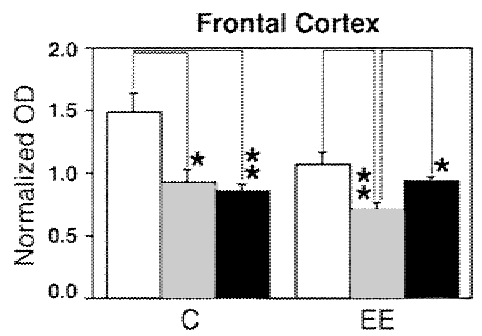

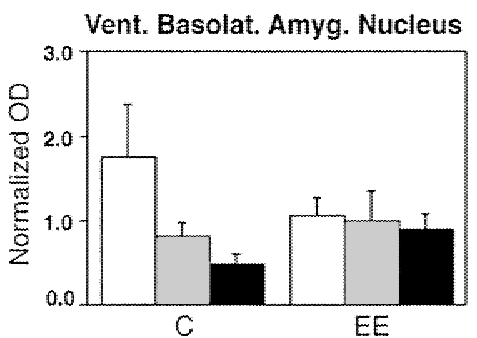

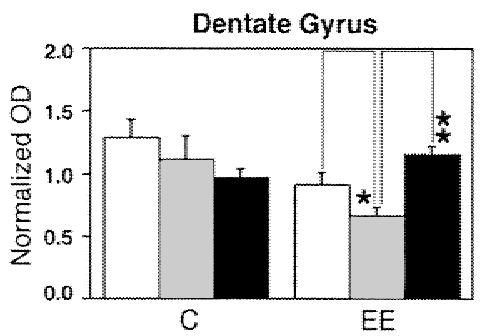

Reuniens Thalamic Nucleus

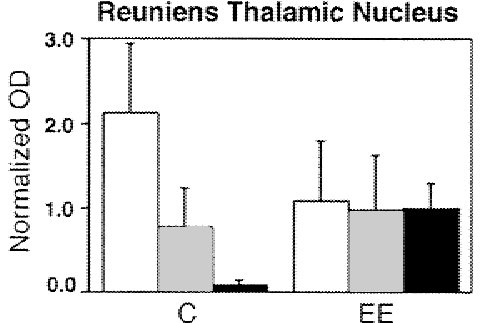

Figure 4: Analysis of zif-268 expression during waking and sleep states in individual brain structures. (Top panels) Three individual structures within the cerebral cortex and the hippocampus in which the most significant differences occurred. Notice that a significant increase in zif-268 expression during REM in EE animals occurred in areas associated with sensory (piriform cortex, $P=0.01$ ), motor (frontal cortex, $P=0.03$ ) and spatial (dentate gyrus, $P=0.03$ ) processing. (Bottom panels) Three individual brain structures in which no significant effect was observed. Ordinates, columns, and asterisks as in Fig. 3.

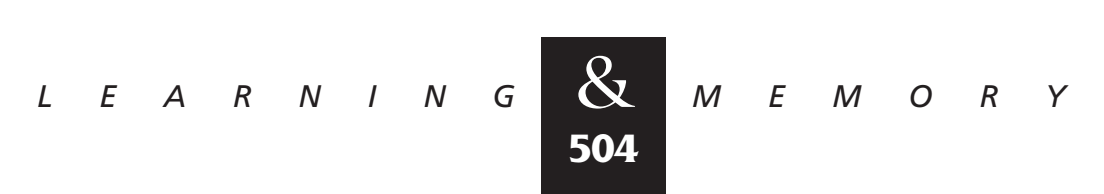


above were not observed in adjacent brain sections hybridized for two other genes encoding zinc-finger transcription factors, krox-20 and ngfi-b (data not shown), and thus appear to be specific for $z i f-268$ within this category of genes.

\section{Discussion}

It has long been known that deprivation of REM sleep-a physiological state characterized by intense cerebral cortex activity, involuntary eye movements, and dreaming (Winson 1993)-impairs short-term or declarative memory in both rats and humans (Pearlman and Becker 1974; Smith and Butler 1982; Hennevin et al. 1995; Fishbein and Gutwein 1977), as well as procedural memories in the latter (Karni et al. 1994). Furthermore, both formal training and exposure to enriched environments have been shown to increase the amount of subsequent REM sleep in rats (Smith 1996). Our study provides evidence showing that brain gene expression during REM sleep depends on previous waking experience.

A brief exposure to an EE had been known to cause a fast and transient induction of various IEGs, including zif-268 (Wallace et al. 1995). The most parsimonious explanation for our present results is that a reinduction of $z i f-268$ occurs in the brain during REM sleep that follows EE exposure. The following supports this interpretation. (1) zif-268 levels in WK did not differ significantly between $\mathrm{C}$ and EE animals. This accords with the well-established transient nature of the IEG response, and argues against the possibility that the high zif-268 expression seen during REM in EE animals stems from a sustained up-regulation (several hours) of zif-268 after the exposure. Also supportive of this view is the fact that REM is always preceded by SW sleep [both in the present study and as reported in the literature (Fishbein and Gutwein 1977; Hennevin et al. 1995; Smith 1996)], and the SW group had consistently low zif-268 levels. (2) In EE animals, the time between exposure to the labyrinth and the moment of sacrifice did not differ among the WK, SW, and REM groups. This rules out the possibility that the high zif-268 expression in the latter represents a putative late wave of gene expression that would be independent of the WK/ sleep state, but simply associated with a longer survival of the REM group after exposure to the labyrinth. (3) The duration of REM sleep did not differ between exposed and control animals. This precludes the possibility that differences in the amount of REM could account for the effects seen. Furthermore, no significant differences in the time spent in SW or WK were found between the REM/C and REM/EE groups (data not shown), discarding the possibility that less SW (or more WK) could explain the up-regulation of zif-268 in REM/ EE animals. (4) For both the cerebral cortex and the hippocampus, zif-268 expression during REM was significantly higher in EE than in C animals. Thus, the high zif-268 levels during REM in the former are not just a reflection of a relative increase with respect to WK and SW levels, but represent a real increase in zif-268 expression during REM from unexposed controls to exposed animals.

The experience provided by exposure to an enriched environment, as in our experimental paradigm, is complex and contains sensory and motor aspects, both of which may have contributed to the gene reinduction observed during REM sleep. Increased motor and somatosensory activities are intrinsic to the exploration of a new environment, and most probably account for a substantial portion of $z i f-268$ reinduction, particularly that occurring in the frontal cortex (Donoghue et al. 1979; Donoghue and Wise 1982). In contrast, the effect observed in the piriform cortex and the hippocampus is likely related to the involvement of these areas in olfaction (Haberly and Price 1978; Schwob et al. 1984) and spatial navigation (Olton et al. 1978; O'Keefe 1993) respectively. Multimodal responses during the exposure period may also have influenced the reinduction pattern described here, as well as increased attentive behavior (increased periods of $\theta$ rhythm). A different experimental design would be necessary to dissect out the specific contributions of the various factors listed above.

The brain expression of $z i f-268$ and other IEGs is highly dependent on the integrity of the noradrenergic system (Cirelli et al. 1996). It has been proposed therefore, that the sleep-related decrease in IEG expression (Pompeiano et al. 1992, 1997; Basheer et al. 1997) reflects the general decrease in neuronal excitability caused by the silencing of the locus coeruleus during sleep (Aston-Jones and Bloom 1981a,b). Such a rationale accounts well for the SW/REM down-regulation of zif-268 observed in our control (unexposed) animals, but not for the gene up-regulation seen during REM sleep in the exposed animals. An intriguing possibility that emerges from our results is that the locus coeruleus is active during REM sleep that follows rich waking experience; to our knowledge, this spe-

$$
\begin{array}{lllllllllllllll}
L & E & A & R & N & I & N & G & \begin{array}{l}
\boldsymbol{Q} \\
\mathbf{5 0 5}
\end{array} & M & E & M & O & R & Y
\end{array}
$$




\section{Ribeiro et al.}

cific possibility has not yet been experimentally tested.

Considering that induced expression of zif-268 depends on neuronal depolarization, our data implies that a rich waking experience affects the brain firing patterns that occur during subsequent REM sleep episodes. This is consistent with the long-held view that the SW/REM structure of sleep is intimately related to previous waking experience (Lucero 1970; Leconte et al. 1974; Ambrosini et al. 1988; Winson 1993; Smith 1996), and attests to the importance of experimentally manipulating such experience to investigate the molecular events associated with REM sleep (Giuditta et al. 1995). The present study constitutes a first demonstration that the expression of an activitydependent gene is up-regulated during REM sleep that follows exposure to an enriched environment. The selective reinduction of zif-268 (but not other zinc-finger IEGs such as $n g f i-b$ or $k r o x-20$ ) allows, in principle, for the regulation of specific subsets of target genes. Given the association between zif268 expression and neuronal plasticity, the phenomenon we described may represent a window of increased plasticity during REM sleep that follows a rich waking experience. Our results thus provide a possible mechanism whereby previous waking experience can contribute to long-lasting changes in the brain.

\section{Acknowledgments}

This work was supported by Kluge and $\mathrm{CNPq} /$ Travel Fellowships (to S.R.), Whitehall Foundation (to C.P), and NIDCD (grant 02853 to C.V.M.). The clones used to derive riboprobes were kindly provided by J. Milbrandt (ngfi-a/zif-268 and ngfi-b) and P. Charnay (krox-20). We thank G. Cecchi, D. Chialvo, D. Herrera, J. Hudspeth, B. McEwen, D. Vicario, and J. Winson for readings of earlier versions of this manuscript; $E$. Jarvis for advice on the behavioral and hybridization protocols; M. Chen and K. Arissian for technical assistance; S. Ogawa and K. Wittkowski for statistical expertise. We also thank Hiroshi Asanuma and Fernando Nottebohm for their continuous support. S.R. dedicates his efforts in this paper to Professor (and dreamer) Isaac Roitman.

The publication costs of this article were defrayed in part by payment of page charges. This article must therefore be hereby marked "advertisement" in accordance with 18 USC section 1734 solely to indicate this fact.

\section{References}

Abraham, W.C., S.E. Mason, J. Demmer, J.M. Williams, C.L. Richardson, and W.P. Tate. 1993. Correlations between immediate early gene induction and the persistence of long-term potentiation. Neuroscience 56: 717-727.
Ambrosini, M.V., A.G. Sadile, C.U. Gironi, M. Mattiaccio, and A. Giuditta. 1988. The sequential hypothesis on sleep function. I. Evidence that the structure of sleep depends on the nature of the previous waking experience. Physiol. Behav. 43: $325-337$.

Aston-Jones, G. and F.E. Bloom. 1981a. Activity of norepinephrine-containing locus coeruleus neurons in behaving rats anticipates fluctuations in the sleep-waking cycle. J. Neurosci. 1: 876-886.

1981b. Norepinephrine-containing locus coeruleus neurons in behaving rats exhibit pronounced responses to non-noxious environmental stimuli. J. Neurosci. 1: 887-900.

Basheer, R., J.E. Sherin, C.B. Saper, J.I. Morgan, R.W. McCarley, and P.J. Shiromani. 1997. Effects of sleep on wake-induced c-fos expression. J. Neurosci. 17: 9746-9750.

Bliss, T.V.P. and G.L. Collingridge. 1993. A synaptic model of memory: Long-term potentiation in the hippocampus. Nature 361: 31-39.

Chaudhuri, A. 1997. Neural activity mapping with inducible transcription factors. NeuroReport 8: 3-7.

Chavrier, P., M. Zerial, P. Lemaire, J. Almendral, R. Bravo, and P. Charnay. 1988. A gene encoding a protein with zinc fingers is activated during G0/G1 transition in cultured cells. EMBO J. 7: 29-35.

Christy, B. and D. Nathans. 1989. DNA binding site of the growth factor-inducible protein Zif268. Proc. Natl. Acad. Sci. 86: $8737-8741$.

Cirelli, C., M. Pompeiano, and G. Tononi. 1996. Neuronal gene expression in the waking state: A role for the locus coeruleus. Science 274: 1211-1215.

Cole, A.J., D.W. Saffen, J.M. Baraban, and P.F. Worley. 1989. Rapid increase of an immediate early gene messenger RNA in hippocampal neurons by synaptic NMDA receptor activation. Nature 340: 474-476.

Cullinan, W.E., J.P. Herman, D.F. Battaglia, H. Akil, and S.J. Watson. 1995. Pattern and time course of immediate early gene expression in rat brain following acute stress. Neuroscience 64: 477-505.

Donoghue, J.P. and S.P. Wise. 1982. The motor cortex of the rat: Cytoarchitecture and microstimulation mapping. J. Comp. Neurol. 212: 76-88.

Donoghue, J.P., K.L. Kerman, and F.F. Ebner. 1979. Evidence for two organizational plans within the somatic sensory-motor cortex of the rat. J. Comp. Neurol. 183: $647-663$.

Fishbein, W. and B.M. Gutwein. 1977. Paradoxical sleep and memory storage processes. Behav. Biol. 19: 425-464.

Giuditta, A., M.V. Ambrosini, P. Montagnese, P. Mandile, M. Cotugno, G.G. Zucconi et al. 1995. The sequential

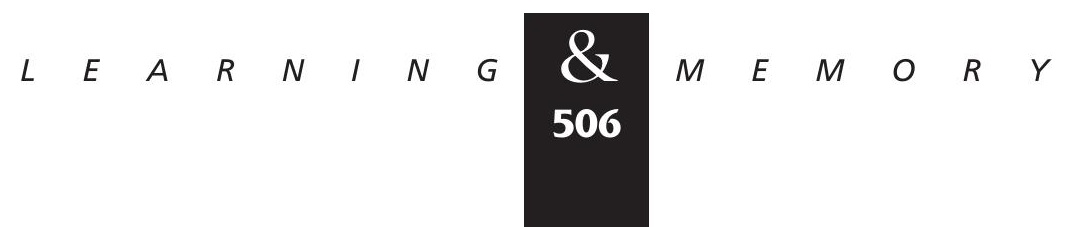


hypothesis of the function of sleep. Behav. Brain Res. 69: 157-166.

Grimm, R. and W. Tischmeyer. 1997. Complex patterns of immediate early gene induction in rat brain following brightness discrimination training and pseudotraining. Behav. Brain Res. 84: 109-116.

Haberly, L.B. and J.L. Price. 1978. Association and commissural fiber systems of the olfactory cortex of the rat. J. Comp. Neurol. 178: 711-740.

Hennevin, E., B. Hars, C. Maho, and V. Bloch. 1995. Processing of learned information in paradoxical sleep: Relevance for memory. Behav. Brain Res. 69: 125-135.

Herdegen, T. and J.D. Leah. 1998. Inducible and constitutive transcription factors in the mammalian nervous system: Control of gene expression by Jun, Fos and Krox, and CREB/ATF proteins. Brain Res. Rev. 28: 370-490.

Honkaniemi, J., S.M. Sagar, I. Pyykonen, K.J. Hicks, and F.R. Sharp. 1995. Focal brain injury induces multiple immediate early genes encoding zinc finger transcription factors. Mol. Brain Res. 28: 157-163.

Jarvis, E.D., C.V. Mello, and F. Nottebohm. 1995. Associative learning and stimulus novelty influence the song-induced expression of an immediate early gene in the canary forebrain. Learn. \& Mem. 2: 62.

Kaczmarek, L. and A. Chaudhuri. 1997. Sensory regulation of immediate-early gene expression in mammalian visual cortex: Implications for functional mapping and neural plasticity. Brain Res. Rev. 23: 237-256.

Kaplan, I.V., Y. Guo, and G.D. Mower. 1995. Developmental expression of the immediate early gene EGR-1 mirrors the critical period in cat visual cortex. Dev. Brain Res. 90: $174-179$.

1996. Immediate early gene expression in cat visual cortex during and after the critical period: Differences between EGR-1 and Fos proteins. Mol. Brain. Res. 36: 12-22.

Karni, A., D. Tanne, B.S. Rubenstein, J.J.M. Askenasy, and D. Sagi. 1994. Dependence on REM sleep of overnight improvement of a perceptual skill. Science 265: 679-682.

Leconte, P., E. Hennevin, and V. Bloch. 1974. Duration of paradoxical sleep necessary for the acquisition of conditioned avoidance in the rat. Physiol. Behav. 13: 675-681.

Lucero, M.A. 1970. Lengthening of REM sleep duration consecutive to learning in the rat. Brain Res. 20: 319-322.

Madison, D.V., R.C. Malenka, and R.A. Nicoll. 1991. Mechanisms underlying long-term potentiation of synaptic transmission. Annu. Rev. Neurosci. 14: 379-397.

Mello, C.V., D.S. Vicario, and D.F. Clayton. 1992. Song presentation induces gene expression in the songbird forebrain. Proc. Natl. Acad. Sci. 89: 6818-6822.

Mello, C., F. Nottebohm, and D. Clayton. 1995. Repeated exposure to one song leads to a rapid and persistent decline in an immediate early gene's response to that song in zebra finch telencephalon. J. Neurosci. 15: 6919-6925.

Milbrandt, J. 1987. A nerve growth factor-induced gene encodes a possible transcriptional regulatory factor. Science 238: 797-799.

1988. Nerve growth factor induces a gene homologous to the glucocorticoid receptor gene. Neuron 1: 183-188.

Nedivi, E., D. Hevroni, D. Naot, D. Israeli, and Y. Citri. 1993. Numerous candidate plasticity-related genes revealed by differential cDNA cloning. Nature 363: 718-722.

Neitz, J. and G.H. Jacobs. 1986. Reexamination of spectral mechanisms in the rat (Rattus norvegicus). J. Comp. Psychol. 100: $21-29$.

Nikolaev, E., B. Kaminska, W. Tischmeyer, H. Matthies, and L. Kaczmarek. 1992. Induction of expression of genes encoding transcription factors in the rat brain elicited by behavioral training. Brain Res. Bull. 28: 479-484.

O'Keefe, J. 1993. Hippocampus, theta, and spatial memory. Curr. Opin. Neurobiol. 3: 917-924.

Olton, D., J.A. Walker, and F.H. Gage. 1978. Hippocampal connections and spatial discrimination. Brain Res. 139: 295-308.

Pavlides, C. and J. Winson. 1989. Influences of hippocampal place cell firing in the awake state on the activity of these cells during subsequent sleep episodes. J. Neurosci. 9: 2907-2918.

Paxinos, G. and C. Watson. 1986. The rat brain in stereotaxic coordinates, Academic Press, New York, NY.

Pearlman, C. and M. Becker. 1974. REM sleep deprivation impairs bar-press acquisition in rats. Physiol. Behav. 13: 813-817.

Petersohn, D., S. Schoch, D.R. Brinkmann, and G. Thiel. 1995. The human synapsin II gene promoter. Possible role for the transcription factor zif268/egr-1, polyoma enhancer activator 3, and AP2. J. Biol. Chem. 270: 24361-24369.

Pompeiano, M., C. Cirelli, and G. Tononi. 1992. Effects of sleep deprivation on fos-like immunoreactivity in the rat brain. Arch. Ital. Biol. 130: 325-335.

Pompeiano, M., C. Cirelli, S. Ronca-Testoni, and G. Tononi. 1997. NGFI-A expression in the rat brain after sleep deprivation. Mol. Brain Res. 46: 143-153.

Richardson, C.L., W.P. Tate, S.E. Mason, P.A. Lawlor, M. Dragunow, and W.C. Abraham. 1992. Correlation between

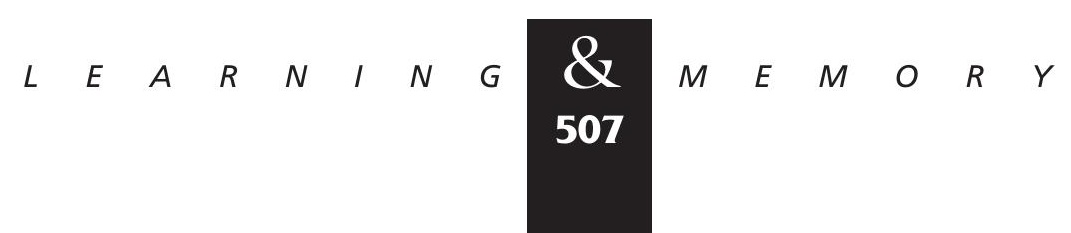




\section{Ribeiro et al.}

the induction of an immediate early gene, zif/268, and long-term potentiation in the dentate gyrus. Brain Res. 580: $147-154$.

Roberts, L.A., M.J. Higgins, C.T. O'Shaughnessy, T.W. Stone, and B.J. Morris. 1996. Changes in hippocampal gene expression associated with the induction of long-term potentiation. Brain Res. Mol. Brain Res. 42: 123-127.

Schwob, J.E., L.B. Haberly, and J.L. Price. 1984. The development of physiological responses of the piriform cortex in rats to stimulation of the lateral olfactory tract. J. Comp. Neurol. 223: 223-237.

Skaggs, W.E. and B.L. McNaughton. 1996. Replay of neuronal firing sequences in rat hippocampus during sleep following spatial experience. Science 271: 1870-1873.

Smith, C. 1996. Sleep states, memory processes and synaptic plasticity. Behav. Brain Res. 78: 49-56.

Smith, C. and S. Butler. 1982. Paradoxical sleep at selective times following training is necessary for learning. Physiol. Behav. 29: 469-473.

Sukhatme, V.P., X.M. Cao, L.C. Chang, C.H. Tsai-Morris, D. Stamenkovich, and P.C. Ferreira et al. 1988. A zinc finger-encoding gene coregulated with c-fos during growth and differentiation, and after cellular depolarization. Cell 53: $37-43$.

Tanaka, M., F. Amaya, Y. Tamada, H. Okamura, Y. Hisa, and Y. Ibata. 1997. Induction of NGFI-A gene expression in the rat suprachiasmatic nucleus by photic stimulation. Brain Res. 756: $305-310$.

Timo-laria, C., R. Yamashita, K. Hoshino, and A. Souza-Melo. 1990. Rostrum movements in desynchronized sleep as a prevalent manifestation of dreaming activity in Wistar rats. Braz. J. Med. Biol. Res. 23: 617-620.

Vanderwolf, C.H. 1969. Hippocampal theta activity and voluntary movement in the rat. Electroenceph. Clin. Neurophysiol. 26: 407-418.

Wallace, C.S., G.S. Withers, I.J. Weiler, J.M. George, D.F. Clayton, and W.T. Greenough. 1995. Correspondence between sites of NGFI-A induction and sites of morphological plasticity following exposure to environmental complexity. Mol. Brain Res. 32: 211-220.

Wilson, M.A. and B.L. McNaughton. 1994. Reactivation of hippocampal ensemble memories during sleep. Science 265: 676-679.

Winson, J. 1974. Patterns of hippocampal theta rhythm in the freely moving rat. Electroenceph. Clin. Neurophysiol. 36: $291-301$

1993. The biology and function of rapid eye movement sleep. Curr. Opin. Neurobiol. 3: 243-248.

Wisden, W., M.L. Errington, S. Williams, S.B. Dunnett, C.
Waters, and D. Hitchcock et al. 1990. Differential expression of immediate early genes in the hippocampus and spinal cord. Neuron 4: 603-614.

Received June 18, 1999; accepted in revised form August 20, 1999.

$$
\begin{array}{lllllllllllllll} 
& E & A & R & N & I & N & G & \mathbf{Q} & M & E & M & 0 & R & Y
\end{array}
$$




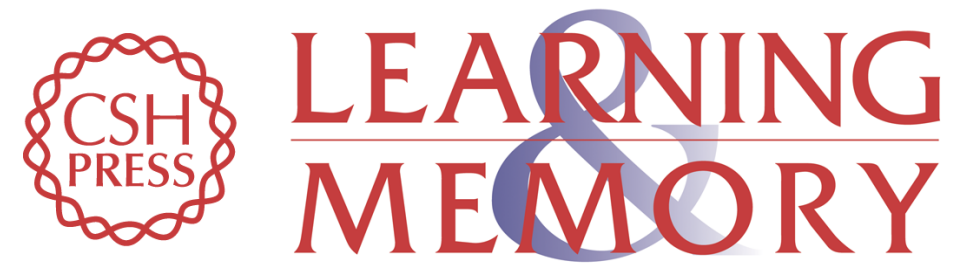

\section{Brain Gene Expression During REM Sleep Depends on Prior Waking Experience}

Sidarta Ribeiro, Vikas Goyal, Claudio V. Mello, et al.

Learn. Mem. 1999, 6:

Access the most recent version at doi:10.1101//m.6.5.500

References This article cites 57 articles, 14 of which can be accessed free at: http://learnmem.cshlp.org/content/6/5/500.full.html\#ref-list-1

License

Email Alerting Receive free email alerts when new articles cite this article - sign up in the box at the Service top right corner of the article or click here. 